\title{
Multiscale Modeling of Thermal Conductivity of High Burnup Structures in $\mathrm{UO}_{2}$ Fuels
}

Xian-Ming Bai ${ }^{*}$, Michael R. Tonks ${ }^{1,2}$, Yongfeng Zhang ${ }^{1}$, and Jason D. Hales ${ }^{1}$

${ }^{1}$ Fuel Modeling and Simulation Department, Idaho National Laboratory, Idaho Falls, ID 83415

${ }^{2}$ Department of Mechanical and Nuclear Engineering, Pennsylvania State University, University Park, PA 16802

*Email: Xianming.Bai@inl.gov

\begin{abstract}
The high burnup structure forming at the rim region in $\mathrm{UO}_{2}$ based nuclear fuel pellets has interesting physical properties such as improved thermal conductivity, even though it contains a high density of grain boundaries and micron-size gas bubbles. To understand this counterintuitive phenomenon, mesoscale heat conduction simulations with inputs from atomistic simulations and experiments were conducted to study the thermal conductivities of a small-grain high burnup microstructure and two large-grain unrestructured microstructures. We concluded that the phonon scattering effects caused by small point defects such as dispersed $\mathrm{Xe}$ atoms in the grain interior must be included in order to correctly predict the thermal transport properties of these microstructures. In extreme cases, even a small concentration of dispersed Xe atoms such as $10^{-5}$ can result in a lower thermal conductivity in the large-grain unrestructured microstructures than in the smallgrain high burnup structure. The high-density grain boundaries in a high burnup structure act as defect sinks and can reduce the concentration of point defects in its grain interior and improve its thermal conductivity in comparison with its largegrain counterparts. An analytical model was developed to describe the thermal conductivity at different concentrations of dispersed Xe, bubble porosities, and grain sizes. Upon calibration, the model is robust and agrees well with independent heat conduction modeling over a wide range of microstructural parameters.
\end{abstract}




\section{Introduction}

Uranium dioxide $\left(\mathrm{UO}_{2}\right)$ is the primary nuclear fuel in today's light water reactors. In service, nuclear fuels are subjected to very harsh irradiation and thermo-mechanical environments. For example, nuclear fission creates significant radiation damage in nuclear fuels. At the normal operating conditions, nuclear fuels receive about one displacement per atom (dpa) per day and a few thousand dpa during the operation lifetime [1]. Fission also creates many different types of fission products. The fission product yield curve as a function of atomic number has two peaks around atomic numbers of 90 and 140 [2]. These fission products can be accommodated as gas bubbles, oxide precipitates, metallic precipitates, or in solid solution in the fuel matrix [3]. Since $\mathrm{UO}_{2}$ has a very low thermal conductivity, a large thermal gradient can build up in the fuels. In light water reactor fuels, the temperature drops by about $700{ }^{\circ} \mathrm{C}$ over $0.5 \mathrm{~cm}$ distance from the fuel pellet center to the rim region [1]. In fast reactors, the thermal gradient is even larger [4]. The thermal gradient can cause strong thermal stresses resulting in cracks, species redistribution, and directional migration of extended defects such as voids [4].

The evolution of both radiation damage defects and fission products in reactor environment results in complex microstructural evolution and thus induces fuel restructuring in nuclear fuels. The microstructure evolution is different at different fuel radial positions $[5,6]$. One of the fuel restructuring phenomena is the formation of high burnup structure (HBS) near the fuel pellet rim region [1]. Before HBS forms, the fuel grain size is about 10 microns and the fuel contains some pores resulting from the fuel fabrication process (Fig. 1(a)). After the HBS forms, the grain size decreases to about $0.1-0.3$ microns through a grain subdivision process. The new grain boundaries (GBs) in HBS are typically low-angle types. At the same time, many micron-size pores or bubbles form. Therefore, HBS has a microstructure of small grains and big bubbles, as shown Fig. 1(b). In light water reactors, formation of HBS starts after about four years of operation. In terms of burnup, HBS starts at a local burnup of about $50 \mathrm{GWd} / \mathrm{tHM}$ and develops fully at about $75 \mathrm{GWd} / \mathrm{tHM}$ [1]. As the burnup increases, HBS extends from the fuel pellet edge towards the pellet center. The increase in width is almost an exponential function of the burnup. At about $100 \mathrm{GWd} / \mathrm{tHM}$, the radial thickness of the HBS is about $1 \mathrm{~mm}$ [7]. The formation of HBS is a complex self-organization process under irradiation and the underlying mechanism is still an open question. The general agreement is that the formation of HBS minimizes the local energy created by radiation (e.g., elastic energy, defect energy, etc.) at the rim region. 

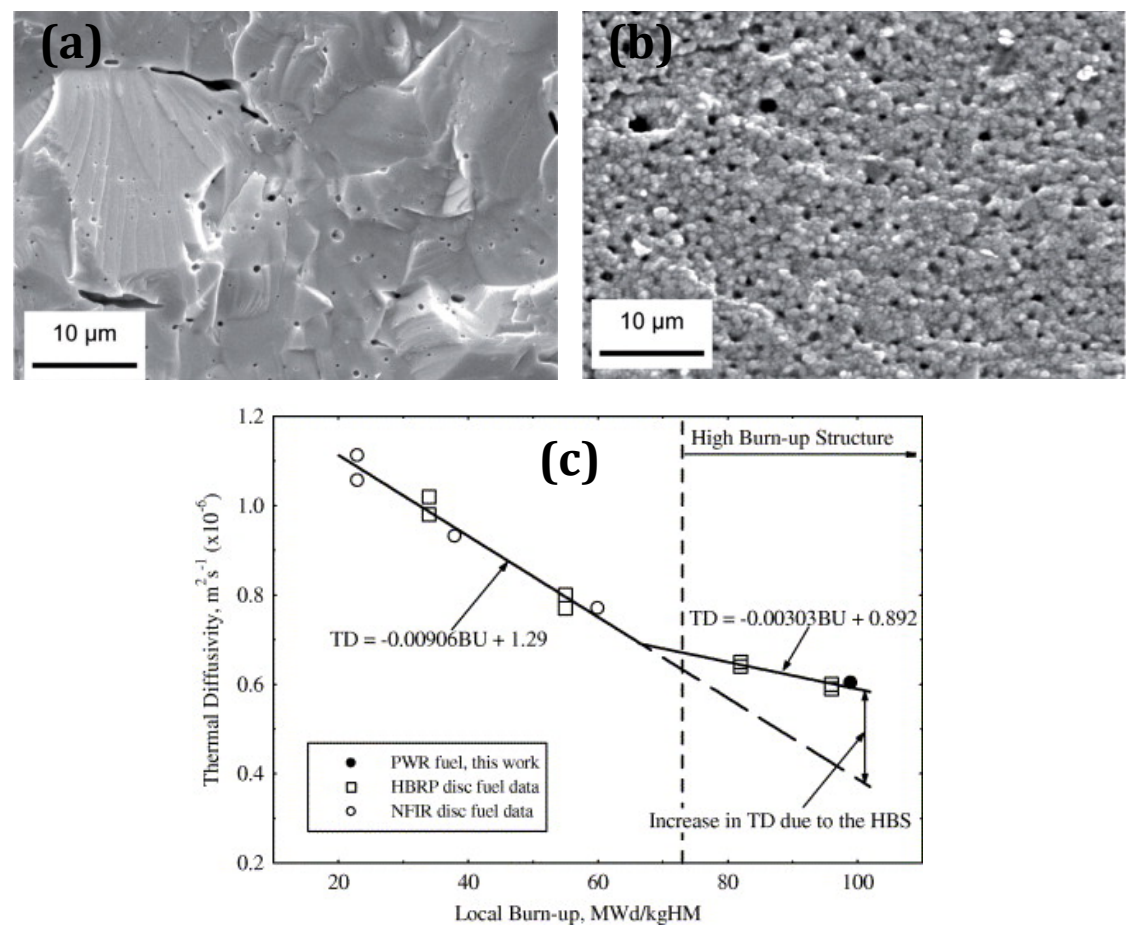

Fig. 1. Experimental results of the microstructure and thermal property of the high burnup structure in $\mathrm{UO}_{2}$ fuels. ( $\mathrm{a}-\mathrm{b}$ ) Scanning electron microscopy (SEM) images of microstructures from fresh fuel and after HBS forms [1]. (c) Thermal diffusivity of $\mathrm{UO}_{2}$ fuels as a function of the fuel burnup. If normalized to the same $95 \%$ of theoretical density, the fuel thermal diffusivity (thus conductivity) after HBS forms is higher than the regression line extrapolated from the low-burnup regime [8]. (Images reproduced with permission.)

Increasing the burnup limit of nuclear fuels can improve the energy production economics, extend the fuel operation time, and reduce the nuclear waste storage [1]. In next-generation reactors, achieving high burnup is also a basic requirement. However, the appearance of HBS in nuclear fuels at high burnups raises safety concerns. Because HBS has a very different microstructure from that at low burnup, the physical properties of HBS also change. To study whether HBS formation impacts the fuel performance negatively, recently researchers have investigated the fission gas release [9], mechanical behavior [10], and thermal conductivity of HBS $[8,11]$. For fission gas release, it was found that the almost all locally generated fission gas was retained in the HBS pores. Therefore, the fission gas retention ability of HBS is a positive feature for fuel performance. The mechanical properties of HBS have been measured by microhardness tests. It was found that the HBS became both softened and toughened. The softened zone may be beneficial for accommodating 
the stress induced by fuel-cladding interaction during fuel swelling. The thermal conductivity of nuclear fuels is a critical physical property for energy conversion efficiency and nuclear safety. GBs have a thermal resistance (i.e., Kapitza resistance [12]) that can cause phonon scattering and reduce the thermal conductivity of fuels. Because HBS contains a high density of GBs, intuitively the thermal conductivity of HBS should be lower than that of the unrestructured microstructure. Interestingly, several studies $[8,11]$ have shown that the reduction of thermal conductivity of the nuclear fuels with the increasing burnup actually is slowed down upon HBS formation (Fig. 1(c)), which is beneficial to the fuel performance. This result is counterintuitive and researchers speculated that the high density of GBs in HBS can annihilate the radiation-induced defects efficiently and reduce the defect concentration in the newly formed subgrains in HBS [1]. However, to date a systematic study to prove this speculation is still lacking.

In this work, we used the MARMOT mesoscale fuel performance tool [13] developed at Idaho National Laboratory with inputs from atomistic modeling and experiments to model the thermal conductivity of HBS. MARMOT predicts the coevolution of microstructure and physical properties in fuel and cladding materials by coupling the phase field method with mechanics and heat conduction. Therefore, the effects of various microstructural features and point defects on thermal transport in nuclear fuels can be studied in MARMOT. We found that the consideration of experimentally-accessible large microstructure features (e.g., grain boundaries and bubbles) alone is not sufficient for interpreting why HBS has an improved thermal conductivity with respect to its unrestructured counterpart at the same porosity. Instead, the phonon scattering effects by dispersed fission gas atoms such as xenon (Xe) must be included in the model. In addition, we also developed an analytical model to predict the thermal conductivity of HBS as a function of different microstructural parameters and the predicted thermal conductivities agree well with independent mesoscale simulation results.

\section{Methodology}

In this work, steady-state heat conduction simulations in MARMOT were used to determine the effective thermal conductivity of various fuel microstructures, where the microstructures were relaxed using phase field simulations. Three microstructures were modeled: large Xe bubbles in small grains (Fig. 2(a)), random small bubbles in large grains (Fig. 2(b)), and GB-aligned small bubbles in large grains (Fig. 2(c)). The first microstructure represents HBS, the second and third ones represent intragranular and intergranular bubbles in unrestructured fuels, 
respectively. The number of grains and bubble radius for each microstructure are shown in Fig. 2.

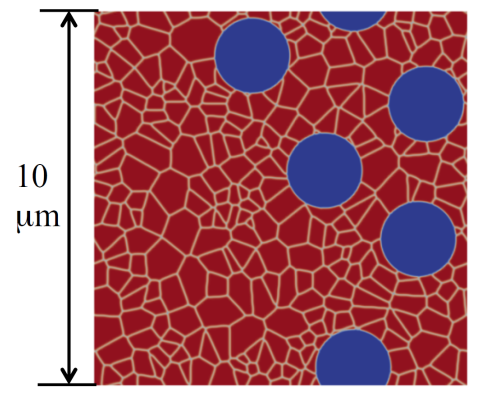

High burnup structures

$\mathrm{N}=300$ grains

Bubble radius $=1 \mu \mathrm{m}$

(a)

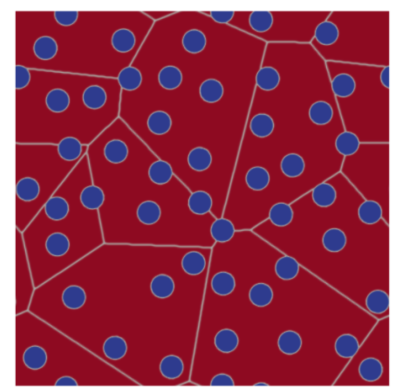

Random bubbles

$\mathrm{N}=10$ grains

Bubble radius $=0.3 \mu \mathrm{m}$

(b)

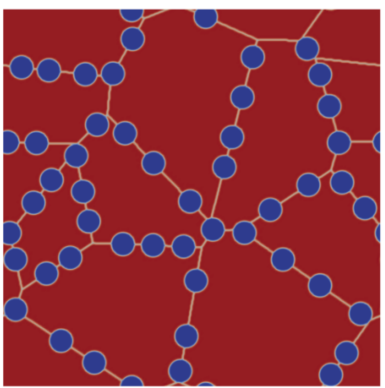

Aligned bubbles

$\mathrm{N}=10$ grains

Bubble radius $=0.3 \mu \mathrm{m}$

(c)

Fig. 2. Three microstructures used for calculating the effective thermal conductivity. (a) A high burnup structure (HBS) that contains small grains and large bubbles. (b) An unrestructured structure with large grains and random small bubbles. (c) An unrestructured structure with large grains and GB-aligned small bubbles. In all figures, the red areas represent the $\mathrm{UO}_{2}$ matrix, the yellow lines represent $\mathrm{GBs}$, and blue circles represent Xe gas bubbles.

All simulations were performed in a 2-D square domain and the domain length was $10 \mu \mathrm{m}$ in each direction (Fig. 2). Assuming each grain has a square geometry, the corresponding grain size is $0.577 \mu \mathrm{m}$ in the 300 -grain system and $3.162 \mu \mathrm{m}$ in the 10-grain systems. The bubble radius is $1 \mu \mathrm{m}$ in HBS and $0.3 \mu \mathrm{m}$ in the large-grain systems, respectively. Note that the bubbles in 2D are in fact represented by cylinders rather than spheres in 3D. It has been shown that at the same porosity cylinders result in more thermal conductivity reduction than spheres [14]. Therefore, caution should be taken if the 2D results are converted to 3D results. The thickness of the diffuse interface in the phase field simulations was set to $50 \mathrm{~nm}$ for both GBs and bubbles. The interfaces are not very smooth at the initial setup so that a short-time phase field simulation was conducted to relax the interfaces for each thermal conductivity calculation. To have fast convergence, the microstructures were relaxed at $1500 \mathrm{~K}$ and no significant grain growth was observed during the relaxation. The Cahn-Hilliard [15] equation was used to model the evolution of bubbles, the Allen-Cahn equation [16] was used to relax the GBs, and the model from Tonks et al. [17] was used for modeling GB-bubble interaction. After the microstructures were relaxed, the thermal conductivity was calculated using Fourier's law at $300 \mathrm{~K}$. Note that at the rim region of $\mathrm{UO}_{2}$ fuel pellets where $\mathrm{HBS}$ 
forms, the temperature is in the range between $673 \mathrm{~K}$ and $973 \mathrm{~K}$ during the normal reactor operation conditions [8]. Although the temperature of $300 \mathrm{~K}$ considered in this work is lower than the relevant temperatures for the HBS formation, the qualitative conclusions from this work are still applicable to the HBS formation temperatures because only phonon-mediated thermal transport mechanism is considered in this work and this mechanism also dominates the thermal transport at the HBS formation temperatures. In the MARMOT simulations, each microstructural feature was assigned with a different thermal conductivity. The thermal conductivity of the $\mathrm{UO}_{2}$ matrix was set to $8.65 \mathrm{~W} / \mathrm{m} \cdot \mathrm{K}$, which corresponds to the experimental thermal conductivity for the fully dense $\mathrm{UO}_{2}$ at $300 \mathrm{~K}$ [18]. The Xe gas bubble thermal conductivity was set to $0.00565 \mathrm{~W} / \mathrm{m} \cdot \mathrm{K}$. The GB local thermal conductivity was set to $5 \mathrm{~W} / \mathrm{m} \cdot \mathrm{K}$, which is equivalent to a Kapitza resistance of 10 $\mathrm{m}^{2} \cdot \mathrm{K} / \mathrm{GW}$ in our simulation setup. Note that currently there is no reported experimental value for the GB Kapitza resistance in $\mathrm{UO}_{2}$ to our best of knowledge. The Kapitza resistance calculated from molecular dynamics simulations ranges from 0.66 to $1.69 \mathrm{~m}^{2} \cdot \mathrm{K} / \mathrm{GW}$ at $300 \mathrm{~K}[19,20]$. These values seem very small, because in other oxides such as $\mathrm{Al}_{2} \mathrm{O}_{3}$ [21, 22], yittria-stabilized $\mathrm{ZrO}_{2}$ [23], and oxygen-deficit $\mathrm{CeO}_{2}$ [24], the reported GB Kapitza resistances in experiments range from 4.5 to 28 $\mathrm{m}^{2} \cdot \mathrm{K} / \mathrm{GW}$. Therefore, our choice of GB Kapitza resistance of $10 \mathrm{~m}^{2} \cdot \mathrm{K} / \mathrm{GW}$ is reasonable in comparison with the experimental results of other oxides. The intrinsic phonon mean free path in $\mathrm{UO}_{2}$ is about $2.6 \mathrm{~nm}$ at $195 \mathrm{~K}$ and it is likely to decrease with the increasing temperature [25]. Clearly, the grain sizes considered in this work $(0.577 \mu \mathrm{m}$ and $3.162 \mu \mathrm{m})$ are much larger than the intrinsic phonon mean free path so that the thermal transport is dominated by multi-phonon scattering events. Therefore, it is reasonable to use the same GB Kapitza resistance for the two different grain sizes in this work. The effective thermal conductivity of the entire system was calculated using the asymptotic expansion homogenization (AEH) method [26] implemented in MARMOT by Hales et al. [27]. In the conventional thermal conductivity calculation method in which a thermal gradient is applied at two domain boundaries, the bubbles falling on the domain boundaries can cause some singularities. The AEH method can avoid this problem and provide more robust results [27].

\section{Results}

The thermal conductivities of the three microstructures shown in Fig. 2 were calculated for different bubble porosities using MARMOT at $300 \mathrm{~K}$. The results are shown in Fig. 3. As expected, the thermal conductivities in all three systems decrease monotonically with increasing bubble porosity $(p)$. The difference in thermal conductivity between the two large-grain systems changes only slightly 
until $p=0.12$. Beyond this porosity, the GB-aligned bubbles have a lower thermal conductivity than the random bubbles, consistent with previous studies [28, 29]. This is because the thermal transport is blocked by the GB-aligned bubbles when the bubble coverage at GBs is high. For both the HBS sample and the large-grain (i.e., unrestructured) sample with random bubbles, the thermal conductivity decreases almost linearly with the increasing porosity. However, over the entire porosity range studied in this work, the HBS has a lower thermal conductivity than that of the unrestructured microstructure with random bubbles. Although the GB-aligned bubbles could have a lower thermal conductivity than HBS, it only happens if GB coverage is very high and GB thermal resistance is small. These results contradict the experimental observation that the local thermal conductivity of the HBS region increases upon its formation with respect to the unrestructured fuel at the same porosity (Fig. 1(c)). Therefore, simply considering the topology of large microstructural features is not sufficient to predict the unusual HBS thermal transport properties.

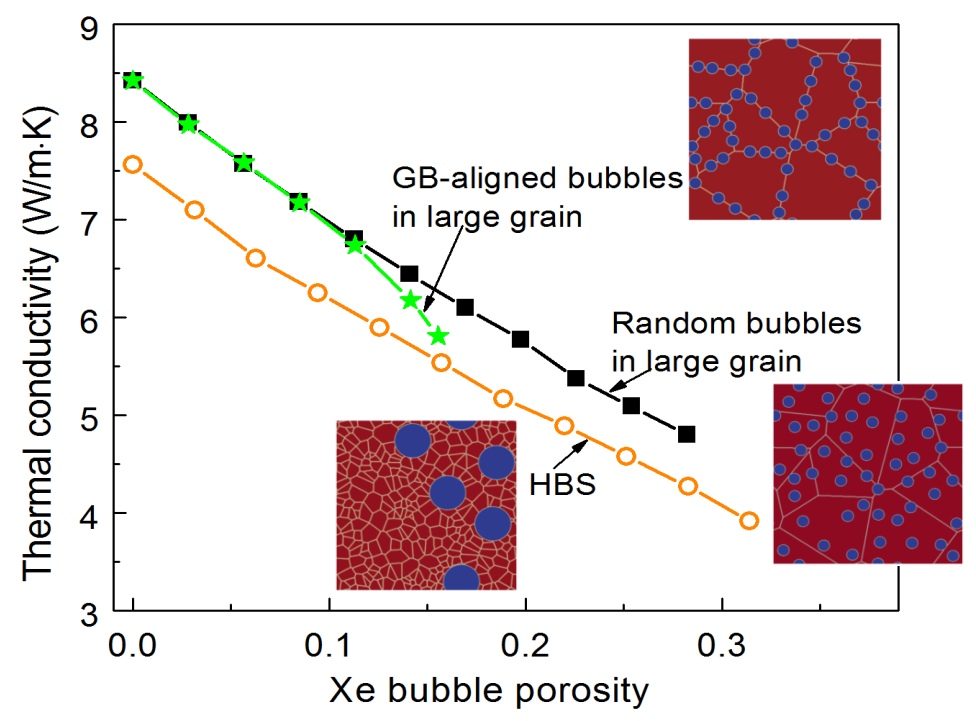

Fig. 3. The thermal conductivities of three microstructures as a function of bubble porosity at $300 \mathrm{~K}$. In these calculations, only the topology of microstructures (e.g., grain size, bubble size and bubble density) is considered.

In reactor environment, nuclear fission induces not only many experimentallyaccessible microstructural defects such as dislocation loops and bubbles, but also many point defects (interstitials, vacancies, dispersed Xe gas atoms, etc.) and small defect clusters. Typically the sizes of these small defects are below the experimental resolution limit so that their concentration is usually not reported. However, when the sizes and their interdistances of these small defects are comparable to the 
intrinsic mean free path of phonons $(\sim$ a few nanometers $[25,30])$, they cause strong phonon scattering effects on thermal transport. A previous study by Teague et al. [31] has shown that if the phonon scattering effect by point defects is neglected in modeling, the thermal conductivity of HBS in a highly irradiated mixed oxide fuel can be even higher than the thermal conductivity in an unirradiated fuel. Therefore, the phonon scattering effects caused by point defects should not be neglected for modeling the thermal conductivity of nuclear fuels.

In this work, the phonon scattering by dispersed Xe atoms is incorporated in the mesoscale modeling. The phonon scattering caused by other types of point defects is a subject of future studies. To include this effect, we incorporated the molecular dynamics (MD) simulation results of the degradation of thermal conductivity at different Xe concentration and temperatures conducted by Tonks et al. [32]. The details of this work were reported elsewhere [32] and here only a brief introduction is provided. In their MD simulations, dispersed Xe atoms were randomly distributed in the $\mathrm{UO}_{2}$ single crystal (SC) matrix. By applying a thermal gradient and using Fourier's law, the thermal conductivities at different Xe concentrations $\left(C_{X e}\right)$ and temperatures $(\mathrm{T})$ were calculated. Based On the well-known temperaturedependence of thermal conductivity (i.e., $\mathrm{k}=1 /(\mathrm{A}+\mathrm{BT})$ ), a Xe-concentrationdependent term is added in the denominator,

$$
k_{S C}(X e, T)=\frac{1}{A+B T+A_{X e} C_{X e}^{n}},
$$

where $A, B, A_{X e}$ and $\mathrm{n}$ are fitting parameters. By fitting to the MD data, $\mathrm{A}=5.77 \times 10^{-2}$ $\mathrm{m} \cdot \mathrm{K} / \mathrm{W}, \mathrm{B}=9.34 \times 10^{-5} \mathrm{~m} / \mathrm{W}, A_{X e}=1.5 \mathrm{~m} \cdot \mathrm{K} / \mathrm{W}$, and $n=0.45$. In Fig. 4(a), the lines show the thermal conductivities predicted by this model as a function of temperature at four different concentrations of dispersed Xe. The symbols represent the thermal conductivities calculated by MD simulations.

However, the empirical potentials in MD simulations typically overpredict the thermal conductivity in $\mathrm{UO}_{2}$ [33]. In addition, the nuclear fuels used in reactors are typically polycrystals with some pores from the fabrication process. Previously, Fink et al. [18] developed an analytical equation to describe the thermal conductivity of polycrystalline $\mathrm{UO}_{2}$ at different temperatures and the model matches with experimental data very well. Here a similar temperature-dependent equation is used for the polycrystalline fuels while an additional Xe-concentration-dependent term is added to the equation,

$$
k_{\text {poly }}(X e, T)=\frac{100}{7.5408+17.692 t+3.6142 t^{2}+100 A_{X e} C_{X e}^{n}}+\frac{6400}{t^{\frac{5}{2}}} \exp \left(\frac{-16.35}{t}\right),
$$


where $t=T(K) / 1000$. The $A_{X e}$ and $n$ values obtained by fitting to the MD data of the single crystal in Eq. (1) were used in Eq. (2). The thermal conductivities of the polycrystal predicted by this model as a function of temperature at four different concentrations of dispersed Xe are shown in Fig. 4(b). Because in Fink's original model the thermal conductivity is for $95 \%$ of the theoretical density, the actual thermal conductivity in MARMOT is scaled to fully dense $\mathrm{UO}_{2}$ using the same scaling law as in Fink's paper [18].
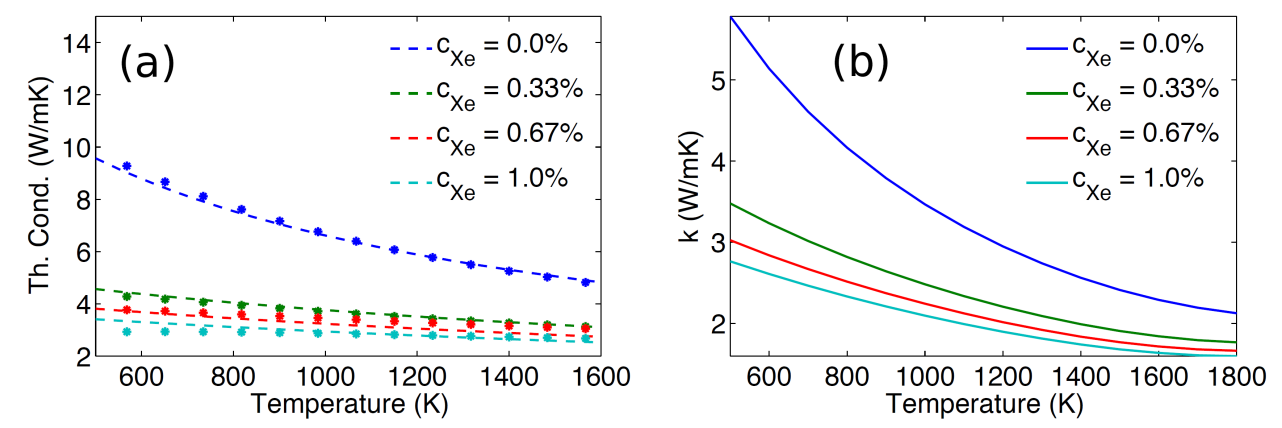

Fig. 4. (a) Thermal conductivities of a single crystal $\mathrm{UO}_{2}$ at four different concentrations of dispersed Xe as a function of temperature. The symbols represent MD simulation data and lines represent the analytical model, Eq. (1). (b) Thermal conductivities of a polycrystal $\mathrm{UO}_{2}$ of $95 \%$ of theory density at four different concentrations of dispersed Xe as a function of temperature predicted by Eq. (2). These plots are taken from a previous work [32].

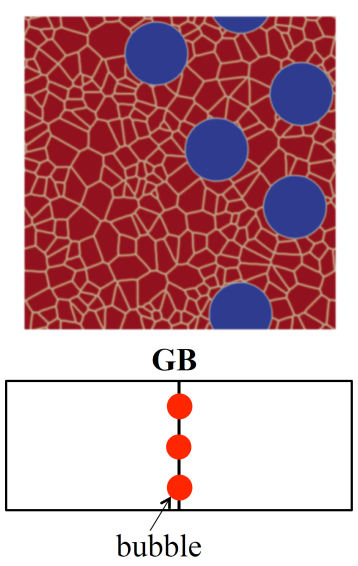

(a)
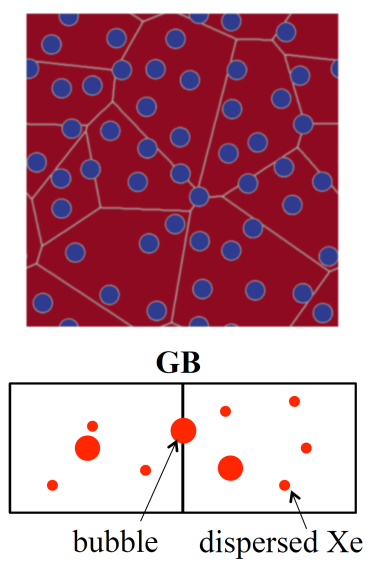

(b)

Fig. 5. Schematic illustration of Xe distribution in HBS and in unrestructured fuels. (a) In HBS no dispersed Xe atoms survive in the grain interior and all Xe atoms form GB bubbles. (b) In an unrestructured fuel of large grains, both dispersed Xe atoms and Xe bubbles are present. 
By implementing the new model (Eq. (2)) in MARMOT, the phonon scattering effect from dispersed Xe atoms can be included in the mesoscale heat conduction simulations. When point defects are present in materials, additional physics need to be considered. It is well known that GBs are defect sinks so that point defects can be removed from the grain interior when the grain size is small [34]. HBS contains a high density of GBs so that the concentration of Xe atoms in the grain interior may be very low. In unrestructured fuels, the grain size is large so that some dispersed Xe atoms can survive in the grain interior if the temperature is not very high. Therefore, as shown in Fig. 5, in our model we assume that in HBS there are no dispersed Xe atoms in the grain interior and all Xe atoms form bubbles; While in the large-grain samples, both Xe bubbles and dispersed Xe atoms are present. Although the assumption of no remaining dispersed Xe atoms in the grain interior of HBS represents an extreme scenario, it is consistent with the speculation by other researchers [1] that HBS can effectively remove point defects from the $\mathrm{UO}_{2}$ matrix and with the experimental observation that the Xe concentration decreases significantly upon HBS formation [35].

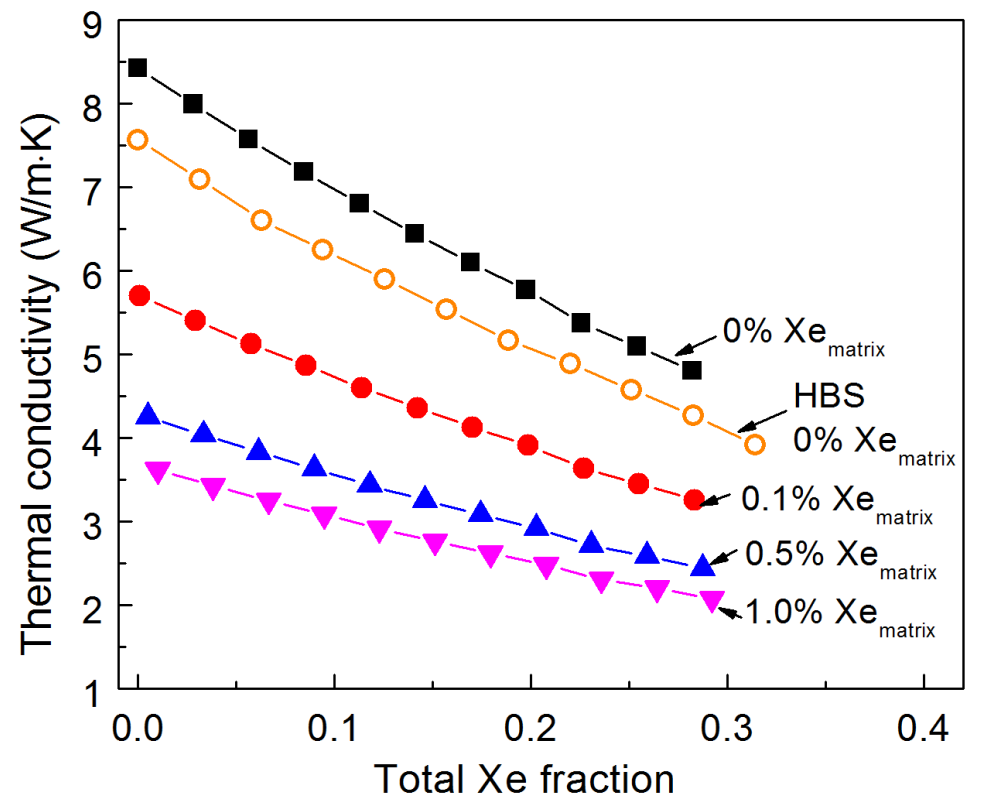

Fig. 6. MARMOT simulation results of the thermal conductivity as a function of total Xe fraction in a $\mathrm{HBS}$ and in a large-grain $\mathrm{UO}_{2}$ with randomly distributed bubbles at $300 \mathrm{~K}$. In HBS no dispersed Xe atoms are present in the grain interior. In the largegrain system the grain interior can contain a small concentration of dispersed Xe atoms as indicated for each data set. 
Figure 6 shows MARMOT simulation results of the thermal conductivity of a HBS (Fig. 5(a)) and a large-grain $\mathrm{UO}_{2}$ with randomly distributed bubbles and dispersed Xe atoms (Fig. 5(b)) as a function of the total Xe fraction at $300 \mathrm{~K}$. The total Xe fraction is defined as the sum of bubble porosity and dispersed Xe concentration. To make this definition, we assume that in the $\mathrm{UO}_{2}$ matrix each Xe atom substitutes a $\mathrm{U}$ lattice site and in bubbles Xe atoms occupy all U lattice sites (i.e., the ratio of Xe atoms to the original $U$ lattice sites is 1.0 in bubbles). In addition, the bubble swelling is neglected. When there are no dispersed Xe atoms present in both systems, the HBS has a lower thermal conductivity than the large-grain system over the entire range of total Xe fraction, which has been shown in Fig. 3. This is because HBS contains many more GBs than the large-grain system and the GBs impede the thermal transport. However, once a small concentration of dispersed Xe is added to the grain interior of the large-grain system, the trend is reversed. As Fig. 6 shows, the large-grain system has a lower thermal conductivity than the HBS at the entire range of total Xe fraction even if only $0.1 \%$ of dispersed Xe atoms are included. The calculations are repeated for $0.5 \%$ and $1 \%$ of dispersed Xe atoms in the large-grain system. As the concentration of dispersed Xe atoms increases, the thermal conductivity of the large-grain system decreases further. Clearly, the incorporation of phonon scattering effects by point defects significantly decreases the thermal conductivity in the large-grain system. On the other hand, the depletion of dispersed Xe atoms by GBs makes the grain interior in HBS free of dispersed-Xe-induced phonon scattering effects. As a result, in HBS the decrease of thermal conductivity caused by GBs can be compensated by the suppression of phonon scattering effects induced by point defects. Therefore, although GBs themselves impede the thermal transport, their interaction with point defects can improve the overall thermal conductivity. This GB-defect interaction could be a very reasonable explanation for why in experiments the thermal conductivity of the HBS region increases with respect to the unrestructured fuels (if both are normalized to $95 \%$ of theoretical density) once the HBS forms (Fig. 1(c)).

Figures 5 and 6 qualitatively explain why a HBS can have a higher thermal conductivity than its large-grain counterpart. In this work, we also try to develop a unified analytical model that can quantitatively predict the thermal conductivity of different microstructures (including HBS) at any given concentration of dispersed Xe, bubble porosity, and grain size. Previously, it has been shown that the effective thermal conductivity of a heterogeneous system can be described by the multiplier of the degradation percentage caused by each type of microstructural feature [36]. In this work a similar approach is adopted. The effective thermal conductivity in a system containing dispersed $\mathrm{Xe}$ in the $\mathrm{UO}_{2}$ matrix, $\mathrm{GBs}$, and bubbles is proposed to be 


$$
k_{X e+G B+b u b b l e}^{e f f}=k_{\text {matrix }} \times \frac{k_{G B}^{e f f}}{k_{\text {matrix }}} \times \frac{k_{\text {bubble }}^{\text {eff }}}{k_{\text {matrix }}},
$$

where $k_{\text {matrix }}$ represents the thermal conductivity of the $\mathrm{UO}_{2}$ matrix. If it contains dispersed Xe atoms, its thermal conductivity $\left(k_{X e}\right)$ can be calculated from Eq. (2) at a given temperature and Xe concentration. For GBs, the widely used polycrystal model [23] is adopted, in which the effective thermal conductivity is related to grain size $D$ and GB Kapitza resistance $R$,

$$
\frac{1}{k_{G B}^{e f f}}=\frac{1}{k_{\text {matrix }}}+\frac{R}{D} .
$$

For voids or bubbles, many different models are available, as summarized by Lee et al. [30]. Here the model proposed by Nikolopoulos et al. [14] is used,

$$
k_{\text {bubble }}^{\text {eff }}=k_{\text {matrix }} \cdot(1-p)^{x},
$$

where $p$ is porosity, $x$ is an exponent and also a fitting parameter. Using Eq. (4), Eq. (5), and $k_{\text {matrix }}=k_{X e}$, Eq. (3) can be rewritten as

$$
k_{X e+G B+b u b b l e}^{\text {eff }}=k_{X e} \times \frac{1}{1+k_{X e} \frac{R}{D}} \times(1-p)^{X} .
$$

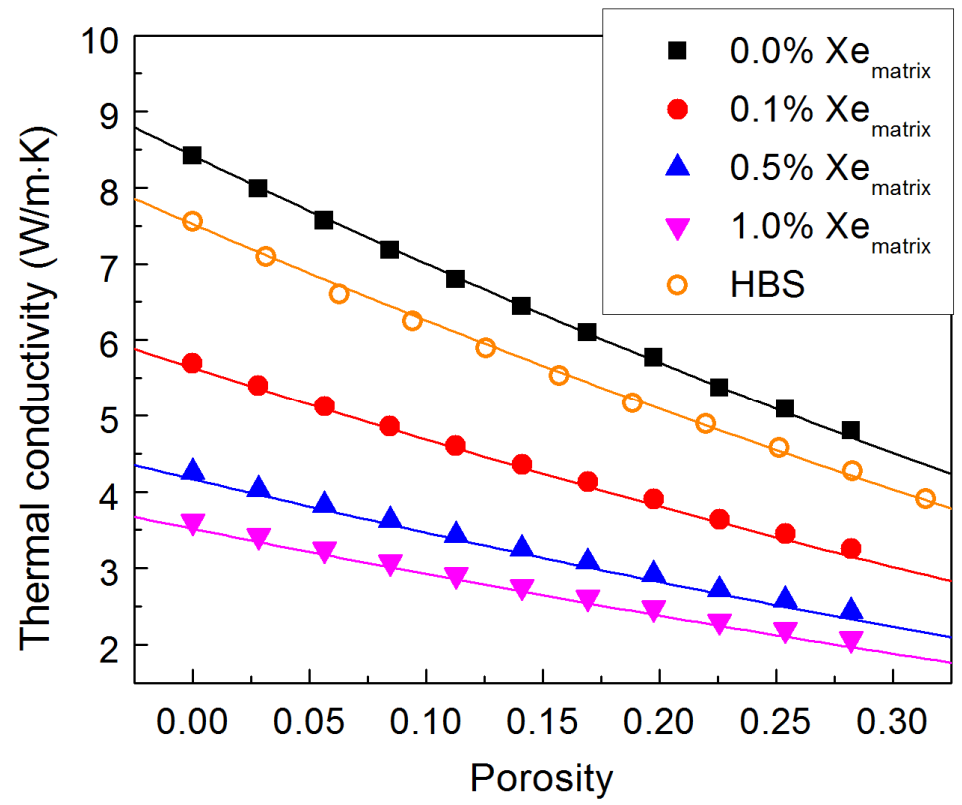

Fig. 7. Comparison between the analytical model (Eq. (6), solid lines) and MARMOT simulation results (symbols) for different microstructural parameters at $300 \mathrm{~K}$. The topmost data set (black squares) is used to calibrate the analytical model so that an 
exponent $x=1.75$ is obtained. The rest of the MARMOT data sets are used to compare with independent model predictions by Eq. (6).

Equation (6) indicates that the effective thermal conductivity is a function of the bubble porosity. Therefore, Figure 6 is replotted as a function of the bubble porosity instead of the total Xe fraction at $300 \mathrm{~K}$, as shown in Fig. 7. To determine the exponent $x$ in Eq. (6), only the thermal conductivities in the large-grain system with $0.0 \%$ dispersed Xe (black squares in Fig. 7) were used to fit to the model. Using the GB Kapitza resistance $\mathrm{R}=10 \mathrm{~m}^{2} \cdot \mathrm{K} / \mathrm{GW}$ and grain size $\mathrm{D}=3.162 \mu \mathrm{m}$, the fitting gives $x=1.75$. After this exponent is determined, the model can predict the thermal conductivity at any given small concentration of dispersed Xe in the matrix, grain size, and porosity without adjusting the exponent. Figure 7 shows the comparison between the model prediction (solid lines) and the MARMOT simulation results (symbols). While the topmost data set is used for calibration, the model prediction is independent of the MARMOT simulation results for all other data points. Clearly, excellent agreement between them can be seen for a wide range of microstructural parameters. In particular, the model correctly predicts the thermal conductivity of HBS that contains larger bubbles and smaller grains. Therefore, the model is very robust and has a predictive power for the conditions beyond its fitting regime.

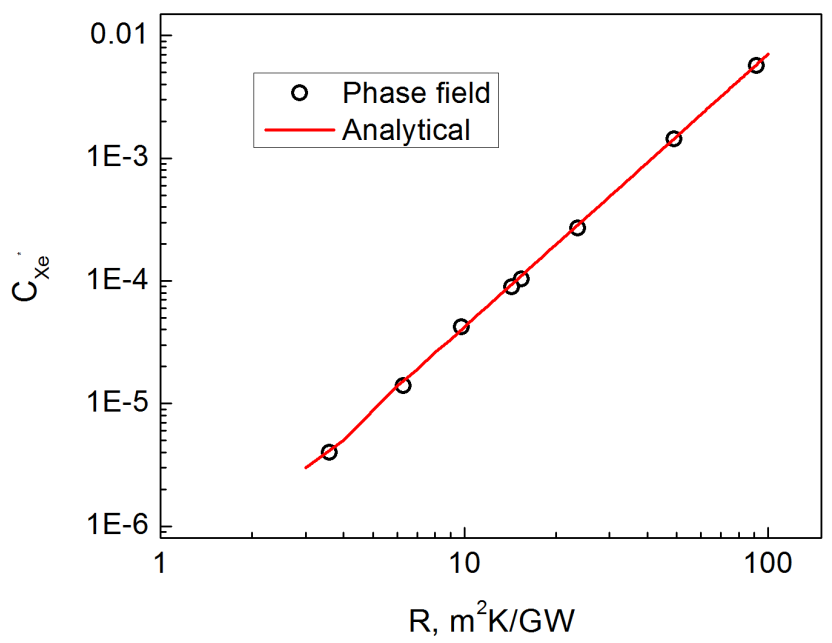

Fig. 8. Critical concentration of dispersed Xe atoms in unrestructured fuels to cause the same thermal conductivity reduction as by GBs in HBS at different GB Kapitza resistances at $300 \mathrm{~K}$. The solid line represents the model prediction (Eq. (7)) and symbols represent independent MARMOT simulation results. 
Figure 7 shows that even $0.1 \%$ of dispersed Xe atoms in the large-grain system can induce more decrease of thermal conductivity than that caused by the high-density GBs in HBS. To estimate the critical concentration of dispersed Xe in an unrestructured fuel that can compensate for the reduction by GBs in HBS, Eq. (6) is used to estimate the thermal conductivity in the unrestructured system (large grains with dispersed Xe) and HBS (small grains without dispersed Xe). By equating their thermal conductivities and assuming that they have the same bubble porosity, an equation can be obtained,

$$
\frac{1}{k_{X e}}+\frac{R}{D_{\text {large }}}=\frac{1}{k_{0}}+\frac{R}{D_{H B S}},
$$

where $k_{0}$ represents the thermal conductivity at $0.0 \%$ dispersed Xe. Using Eq. (2) to estimate $k_{X e}$ and $k_{0}$, and $D_{\text {large }}=3.162 \mu \mathrm{m}$ and $D_{H B S}=0.577 \mu \mathrm{m}$, Eq. (7) is numerically solved to give a correlation between the critical concentration of dispersed Xe in large-grain system $\left(\mathrm{C}_{\mathrm{Xe}}{ }^{*}\right)$ and the GB Kapitza resistance (R). The numerical solution of Eq. (7) is plotted as the solid line in Fig. 8. In the log-log plot, $\mathrm{C}_{\mathrm{Xe}}{ }^{*}$ increases almost linearly with R. To check the validity of Eq. (7), spot checks using MARMOT simulations were performed at different Kapitza resistances to determine the corresponding $\mathrm{CXe}^{*}$. The MARMOT results are shown as symbols in Fig. 8. Clearly, there is excellent agreement between the analytical model (Eq. (7)) and the independent MARMOT calculations. The good agreement suggests that Eq. (7) can be used to estimate the correlation between the critical Xe concentration and GB Kapitza resistance if the grain sizes in unrestructured and restructured fuels are known. For example, for the grain sizes used in this work, a concentration of about $\mathrm{CXe}^{*}=3 \times 10^{-5}$ of dispersed Xe atoms in the unrestructured fuels $\left(D_{\text {large }}=3.162\right.$ $\mu \mathrm{m})$ can cause the same reduction of thermal conductivity in $\operatorname{HBS}\left(D_{H B S}=0.577 \mu \mathrm{m}\right)$ for a typical Kapitza resistance of $R=10 \mathrm{~m}^{2} \cdot \mathrm{K} / \mathrm{GW}$.

The above analysis is for an ideal scenario in which all the dispersed Xe atoms are removed from the grain interior of HBS. In reality, there are still some dispersed Xe atoms remaining in the grain interior of HBS. Previously, some researchers used electron probe microanalysis (EPMA) to measure the local concentration of Xe at the fuel rim region as a function of fuel burnup [35]. The work showed that the local Xe concentration reached about $1.1 \mathrm{wt} . \%(\sim 2.2$ at.\%) right before the HBS started to form and decreased to about 0.3 wt.\% ( $\sim 0.6$ at.\%) after the HBS formed completely. Using our thermal conductivity model for the full dense $\mathrm{UO}_{2}$, the corresponding thermal conductivity of the fuel matrix is about $2.842 \mathrm{~W} / \mathrm{m} \cdot \mathrm{K}$ and $4.044 \mathrm{~W} / \mathrm{m} \cdot \mathrm{K}$ at these Xe concentrations, respectively. If the same grain sizes and Kapitza resistance shown in the previous paragraph are used, the HBS still results in a higher thermal conductivity than the large grain system at the same porosity. This result suggests 
that according to our model the impact of dispersed Xe alone is sufficient to explain the beneficial effect of the HBS formation for the thermal transport.

\section{Discussion and Conclusions}

In this work, mesoscale heat conduction simulations were used to study the unusual thermal transport properties in the $\mathrm{HBS}$ of $\mathrm{UO}_{2}$ fuels, which contains large bubbles and small grains. For comparison, the unrestructured microstructures in $\mathrm{UO}_{2}$ fuels were also studied, which contain small intergranular or intragranular bubbles and large grains. Our results show that the thermal conductivity of HBS is lower than the unrestructured microstructure if only the topology of different meso-scale microstructural features (e.g., GBs and bubbles) is considered, contradicting the experimental observation. We concluded that the phonon scattering effects caused by point defects such as dispersed Xe atoms in the matrix should not be neglected in the model. Using the results from molecular dynamics simulations as input, the thermal conductivities at different concentrations of dispersed Xe atoms and temperatures were incorporated in the mesoscale modeling. Using the new thermal conductivity model, the correct trend that HBS has a higher thermal conductivity than unrestructured microstructures was successfully predicted, because the highdensity GBs in HBS could make its grain interior free of dispersed Xe atoms while the low-density GBs in unrestructured microstructures result in a small amount of dispersed Xe atoms in the grain interior. Our results show that in an ideal scenario even a small concentration of dispersed Xe atoms (e.g., 10-5) in the grain interior can offset the reduction of thermal conductivity by GBs in HBS. Therefore, this work provides a reasonable explanation for the counterintuitive experimental observation that the thermal conductivity of the rim region in $\mathrm{UO}_{2}$ fuels increases after HBS forms.

To quantitatively predict the thermal conductivity of $\mathrm{UO}_{2}$ at different concentrations of dispersed Xe atoms, bubble porosities, and grain sizes, a multiplier model was adopted. In this model, the thermal conductivity of a heterogeneous microstructure can be described by multiplying the reduction ratio of each unit mechanism. The thermal conductivities predicted by this analytical model agree with our independent mesoscale simulations very well for different microstructural parameters, including HBS. Although in this work only grain size, concentration of dispersed Xe atoms, Xe bubble porosity were considered in the multiplier model, the model can be extended to including additional defect types. In nuclear fuels, many other defects can affect the thermal conductivity such as interstitials and vacancies, other fission products, metallic precipitates, etc. These defects have not been studied in this work but will be a topic of future research. If each unit reduction mechanism of these defects is understood, the multiplier model can be conveniently applied to incorporate them to predict the thermal conductivity of nuclear fuels. 
In HBS, the average bubble size is typically larger than that in the unrestructured fuels. At the same porosity, the increase of bubble size reduces the total bubble surface area thus the phonon-surface interaction probability. It has been shown that by tuning the size of solid particles embedded in a solid matrix the effective thermal conductivity can change significantly $[37,38]$. Therefore, the observed higher HBS conductivity relative to that of the unrestructured fuels at the same porosity could be a result of the decreased surface area. In this work, the phonon scattering at the bubble surfaces was not taken into account but it can be a future research topic.

In this work, all the mesoscale simulations of thermal conductivity were performed in 2D. In reality, the thermal conductivities are measured in 3D. For simple and ideal microstructures, it has been shown that the 2-D results can be converted to 3D data by multiplying geometric factors [39]. For such cases, simulations may be conducted in 2-D to save the computational cost. However, for complex microstructures the conversion from $2 \mathrm{D}$ to $3 \mathrm{D}$ is not straightforward. Therefore, developing the capability for calculating the thermal conductivity of complex microstructures in 3D is still very important.

\section{Acknowledgements}

The authors would like to acknowledge discussions with Dr. Christopher Stanek from Los Alamos National Laboratory that motivated this work. The authors also would like to acknowledge Dr. Xiang-Yang Liu and Dr. David Andersson at Los Alamos National Laboratory for sharing their atomistic data and having helpful discussion. This work is supported by the U.S. Department of Energy, Office of Nuclear Energy, Nuclear Energy Advanced Modeling and Simulation (NEAMS) Program. This manuscript has been authored by Battelle Energy Alliance, LLC under Contract No. DE-AC07-05ID14517 with the U.S. Department of Energy. The United States Government retains and the publisher, by accepting the article for publication, acknowledges that the United States Government retains a nonexclusive, paid-up, irrevocable, world-wide license to publish or reproduce the published form of this manuscript, or allow others to do so, for United States Government purposes.

\section{References}

[1] V. V. Rondinella, and T. Wiss, The high burn-up structure in nuclear fuel, Materials Today 13, 24-32 (2010). 
[2] http://t2.lanl.gov/nis/tour/sch007.html.

[3] H. Kleykamp, The chemical state of the fission products in oxide fuels, Journal of Nuclear Materials 131, 221-246 (1985).

[4] D. R. Olander, Fundamental Aspects of Nuclear Reactor Fuel Elements (Technical Information Center, Office of Public Affairs, Energy Research and Development Administration, 1976).

[5] H. Stehle, Performance of oxide nuclear fuel in water-cooled power reactors, Journal of Nuclear Materials 153, 3-15 (1988).

[6] J. Noirot, L. Desgranges, and J. Lamontagne, Detailed characterisations of high burn-up structures in oxide fuels, Journal of Nuclear Materials 372, 318-339 (2008).

[7] R. Manzel, and C. T. Walker, EPMA and SEM of fuel samples from PWR rods with an average burn-up of around $100 \mathrm{MWd} / \mathrm{kgHM}$, Journal of Nuclear Materials 301, 170-182 (2002).

[8] C. T. Walker, D. Staicu, M. Sheindlin, D. Papaioannou, W. Goll, and F. Sontheimer, On the thermal conductivity of UO2 nuclear fuel at a high burn-up of around 100MWd/kgHM, Journal of Nuclear Materials 350, 19-39 (2006).

[9] J. P. Hiernaut, T. Wiss, J. Y. Colle, H. Thiele, C. T. Walker, W. Goll, and R. J. M. Konings, Fission product release and microstructure changes during laboratory annealing of a very high burn-up fuel specimen, Journal of Nuclear Materials 377, 313-324 (2008).

[10] J. Spino, J. Cobos-Sabate, and F. Rousseau, Room-temperature microindentation behaviour of LWR-fuels, part 1: fuel microhardness, Journal of Nuclear Materials 322, 204-216 (2003).

[11] C. Ronchi, M. Sheindlin, D. Staicu, and M. Kinoshita, Effect of burn-up on the thermal conductivity of uranium dioxide up to 100.000 MWdt-1, Journal of Nuclear Materials 327, 58-76 (2004).

[12] P. L. Kapitza, The study of heat transfer in helium II, Journal of Physics (USSR) 4, 181 - 210 (1941).

[13] M. R. Tonks, D. Gaston, P. C. Millett, D. Andrs, and P. Talbot, An objectoriented finite element framework for multiphysics phase field simulations, Computational Materials Science 51, 20-29 (2012).

[14] P. Nikolopoulos, and G. Ondracek, Conductivity bounds for porous nuclear fuels, Journal of Nuclear Materials 114, 231-233 (1983).

[15] J. W. Cahn, and J. E. Hilliard, Free Energy of a Nonuniform System. I. Interfacial Free Energy, The Journal of Chemical Physics 28, 258-267 (1958).

[16] S. M. Allen, and J. W. Cahn, A microscopic theory for antiphase boundary motion and its application to antiphase domain coarsening, Acta Metallurgica 27, 1085-1095 (1979). 
[17] R. T. Michael, Z. Yongfeng, B. Aaron, and B. Xian-Ming, Development of a grain boundary pinning model that considers particle size distribution using the phase field method, Modelling and Simulation in Materials Science and Engineering 23, 045009 (2015).

[18] J. K. Fink, Thermophysical properties of uranium dioxide, Journal of Nuclear Materials 279, 1-18 (2000).

[19] M. R. Tonks, P. C. Millett, P. Nerikar, S. Du, D. Andersson, C. R. Stanek, D. Gaston, D. Andrs, and R. Williamson, Multiscale development of a fission gas thermal conductivity model: Coupling atomic, meso and continuum level simulations, Journal of Nuclear Materials 440, 193-200 (2013).

[20] T. Chen, D. Chen, B. H. Sencer, and L. Shao, Molecular dynamics simulations of grain boundary thermal resistance in UO2, Journal of Nuclear Materials 452, 364-369 (2014).

[21] K. Tai, A. Lawrence, M. P. Harmer, and S. J. Dillon, Misorientation dependence of Al2O3 grain boundary thermal resistance, Applied Physics Letters 102, 034101 (2013).

[22] D. S. Smith, S. Fayette, S. Grandjean, C. Martin, R. Telle, and T. Tonnessen, Thermal Resistance of Grain Boundaries in Alumina Ceramics and Refractories, Journal of the American Ceramic Society 86, 105-111 (2003).

[23] H.-S. Yang, G. R. Bai, L. J. Thompson, and J. A. Eastman, Interfacial thermal resistance in nanocrystalline yttria-stabilized zirconia, Acta Materialia 50, 23092317 (2002).

[24] M. Khafizov, I.-W. Park, A. Chernatynskiy, L. He, J. Lin, J. J. Moore, D. Swank, T. Lillo, S. R. Phillpot, A. El-Azab, and D. H. Hurley, Thermal Conductivity in Nanocrystalline Ceria Thin Films, Journal of the American Ceramic Society 97, 562-569 (2014).

[25] J. P. Moore, and D. L. McElroy, Thermal Conductivity of Nearly Stoichiometric Single-Crystal and Polycrystalline UO2, Journal of the American Ceramic Society 54, 40-46 (1971).

[26] V. V. Jikov, S. M. Kozlov, and O. A. Oleinik, Homogenization of Differential Operators and Integral Functionals (Springer-Verlag, New York, 1994).

[27] J. D. Hales, M. R. Tonks, K. Chockalingam, D. M. Perez, S. R. Novascone, B. W. Spencer, and R. L. Williamson, Asymptotic expansion homogenization for multiscale nuclear fuel analysis, Computational Materials Science 99, 290-297 (2015).

[28] K. Chockalingam, P. C. Millett, and M. R. Tonks, Effects of intergranular gas bubbles on thermal conductivity, Journal of Nuclear Materials 430, 166-170 (2012).

[29] P. C. Millett, and M. Tonks, Meso-scale modeling of the influence of intergranular gas bubbles on effective thermal conductivity, Journal of Nuclear Materials 412, 281-286 (2011). 
[30] C. W. Lee, A. Chernatynskiy, P. Shukla, R. E. Stoller, S. B. Sinnott, and S. R. Phillpot, Effect of pores and He bubbles on the thermal transport properties of UO2 by molecular dynamics simulation, Journal of Nuclear Materials 456, 253259 (2015).

[31] M. Teague, B. Fromm, M. Tonks, and D. Field, Using Coupled Mesoscale Experiments and Simulations to Investigate High Burn-Up Oxide Fuel Thermal Conductivity, JOM Journal of the Minerals, Metals and Materials Society 66, 2569-2577 (2014).

[32] M. R. Tonks, X.-Y. Liu, D. Andersson, D. Perez, A. Chernatynskiy, G. Pastore, C. R. Stanek, and R. Williamson, Development of a multiscale thermal conductivity model for fission gas in UO2, Journal of Nuclear Materials 469, 8998 (2016).

[33] A. Chernatynskiy, C. Flint, S. Sinnott, and S. Phillpot, Critical assessment of UO2 classical potentials for thermal conductivity calculations, Journal of Materials Science 47, 7693-7702 (2012).

[34] X.-M. Bai, A. F. Voter, R. G. Hoagland, M. Nastasi, and B. P. Uberuaga, Efficient Annealing of Radiation Damage Near Grain Boundaries via Interstitial Emission, Science 327, 1631-1634 (2010).

[35] C. Walker, Electron probe microanalysis of irradiated nuclear fuel: an overview, Journal of Analytical Atomic Spectrometry 14, 447-454 (1999).

[36] P. C. Millett, D. Wolf, T. Desai, S. Rokkam, and A. El-Azab, Phase-field simulation of thermal conductivity in porous polycrystalline microstructures, Journal of Applied Physics 104, 033512 (2008).

[37] N. Mingo, D. Hauser, N. P. Kobayashi, M. Plissonnier, and A. Shakouri, "Nanoparticle-in-Alloy" Approach to Efficient Thermoelectrics: Silicides in SiGe, Nano Letters 9, 711-715 (2009).

[38] H. Zhang, and A. J. Minnich, The best nanoparticle size distribution for minimum thermal conductivity, Scientific Reports 5, 8995 (2015).

[39] K. Bakker, Using the finite element method to compute the influence of complex porosity and inclusion structures on the thermal and electrical conductivity, International Journal of Heat and Mass Transfer 40, 3503-3511 (1997). 